\title{
Pathogenesis of Alfalfa mosaic virus in Soybean (Glycine max) and Expression of Chimeric Rabies Peptide in Virus-Infected Soybean Plants-A Reexamination
}

\author{
Robert Brodzik, Deepali Deka, Alexander Karasev, and Hilary Koprowski
}

The Biotechnology Foundation at Thomas Jefferson University, 1020 Locust Street, Philadelphia, PA 19107.

Accepted for publication 5 August 2002.

A paper entitled "Pathogenesis of Alfalfa mosaic virus in Soybean (Glycine max) and Expression of Chimeric Rabies Peptide in Virus-Infected Soybean Plants" by Nina Fleysh, Deepali Deka, Maria Drath, Hilary Koprowski, and Vidadi Yusibov was published recently in Phytopathology (1). Two claims were made in the paper: (i) Soybean can be successfully infected with wild Alfalfa mosaic virus (AIMV), and (ii) recombinant of AIMV coat protein fused to rabies virus peptide could infect soybean.

In trying to extend the data presented in the paper, we were unable, using the same rabies construct, to confirm the expression of rabies epitope in the soybeans. We performed exact replicas of the described experiments to reexamine the results.

The plasmid clones pUT17, pUT27, pALg24, and A4 (1) were from the laboratory collection. Plasmids were linearized with the restriction enzymes SmaI, PstI, PstI, and SmaI, respectively, and transcribed with the T7 RNA polymerase (pUT17, pUT27, and pALg24) or SP6 RNA polymerase (A4) in vitro in the presence of the cap-analogue as described (1). The four RNA transcripts were inoculated into soybean as described (1) and symptom development was monitored 1 to 4 weeks postinoculation; at least three plants were used per sample for inoculations. To confirm the status of the inoculated plants, leaf tissue was sampled at different times postinoculation and tested in immunoblots (1) using AlMVspecific antibodies (Agdia, Elkhart, IN). As a positive control for the quality of transcription, an NFlg24 clone of the AIMV RNA3 was processed in parallel, i.e., the NFlg24 clone was linearized with $\mathrm{SmaI}$ and used for transcription with T7 RNA polymerase in the presence of the cap-analogue. The resulting NFlg24 transcript was inoculated to P12 Nicotiana tabacum plants that can support the replication of the RNA3 of AIMV (Fig. 1A). The development of symptoms in P12 plants was monitored visually 1 to 3 weeks postinoculation and confirmed by immunoblotting (Fig. 1A). In control experiments, AIMV was inoculated into soybean plants as described and symptom development and virus accumulation was monitored 1 to 4 weeks postinoculation (Fig. 1B).

The results of the testing of recombinant, capped transcripts in soybean and $N$. benthamiana plants are summarized in Table 1. The capped transcripts of pUT17, pUT27, pALg24, and A4 did not produce visible symptoms in soybean or $N$. benthamiana upon inoculation. The lack of productive infection was confirmed by western blots of the lower inoculated leaves as well as upper uninoculated leaves (Table 1). The control, capped transcript NFlg24 resulted in normal productive infection upon inoculation into the P12 tobacco plants with clearly visible local and systemic

Corresponding author: H. Koprowski; E-mail address: h_koprowski@lac.jci.tju.edu

Publication no. P-2002-1016-010

(c) 2002 The American Phytopathological Society

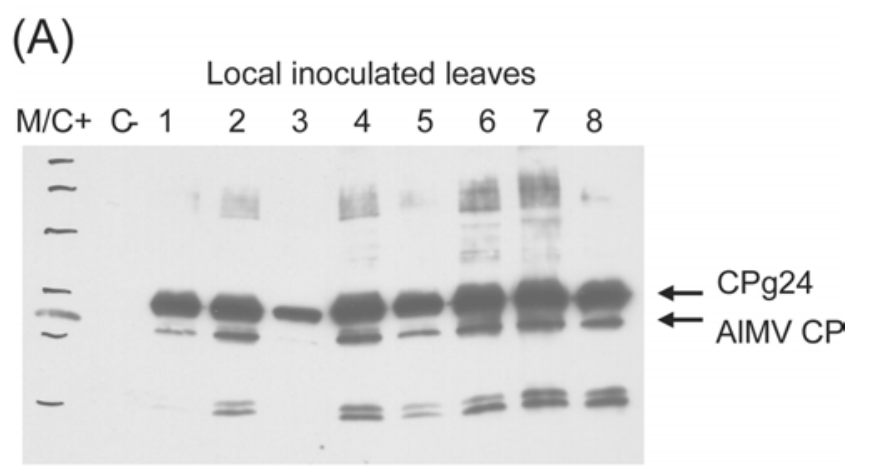

Systemic not inoculated leaves

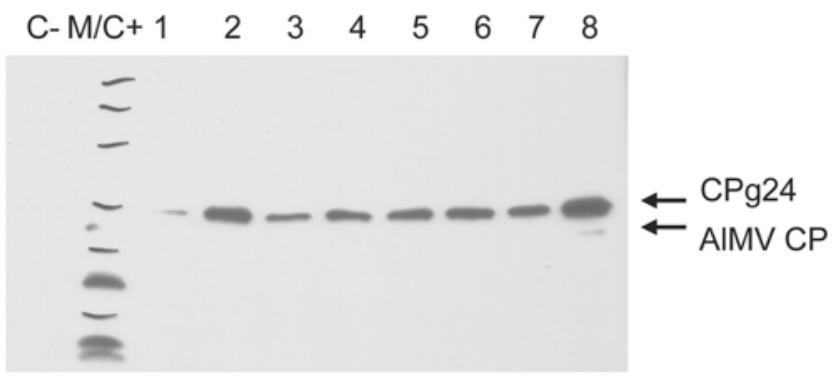

(B)

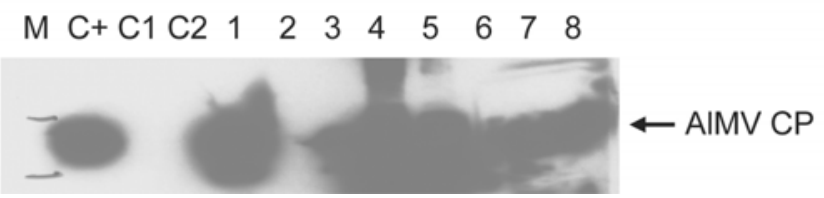

Fig. 1. Western blot analysis of A, Nicotiana tabacum P12 plants inoculated with NF1g24 transcript and B, soybean and $N$. benthamiana plants inoculated with wild type of Alfalfa mosaic virus (AIMV). Proteins were separated electophoretically on $12 \%$ sodium dodecyl sulfate-polyacrylamide gel, transferred to a nitrocellulose membrane, and exposed to monoclonal antibodies specific for AlMV coat protein (CP) as described in literature citation 1. Samples were obtained from infected plants at 8 days postinoculation. A, Numbers 1 to 8 represent separate plants of $N$. tabacum P12. B, Lanes 1 and 2 represent soybean (local leaves); or lanes 3, 4, and 5 (local leaves), and lanes 6,7 , and 8 (systemic leaves) represent $N$. benthamiana separate plants infected with AlMV. Lane $\mathrm{M}$ is Bio-Rad broad-range prestained protein marker (Bio-Rad Laboratories, Hercules, CA): from top A, 209, 124, 80, 49, $34.8,28.9$, and $20.6 \mathrm{kDa}$ and $\mathbf{B}, 34.8$ and $28.9 \mathrm{kDa}$; $\mathbf{C}+$ is purified AlMV; $\mathbf{A}$, $\mathrm{C}$ - is mock-inoculated P12 plant; and $\mathbf{B}, \mathrm{C} 1$ is mock-inoculated soybean and $\mathrm{C} 2$ is $N$. benthamiana plant. 
TABLE 1. Inoculation of the Alfalfa mosaic virus transcripts in Glycine max (soybean) and Nicotiana benthamiana plants

\begin{tabular}{|c|c|c|c|c|c|}
\hline \multirow[b]{2}{*}{ Experiment no. } & \multirow[b]{2}{*}{ Transcripts used for inoculation } & \multicolumn{2}{|c|}{ G. $\max$ (soybean) } & \multicolumn{2}{|c|}{ N. benthamiana } \\
\hline & & Ratio of infection & Immunoblot & Ratio of infection & Immunoblot \\
\hline 1 & pUT17, pUT27, pALg24, and A4 & $0 / 12$ & Negative & $0 / 12$ & $\mathrm{NT}^{\mathrm{a}}$ \\
\hline
\end{tabular}

${ }^{\mathrm{a}} \mathrm{NT}=$ not tested.

reaction, confirmed by immunoblotting of inoculated leaves and upper uninoculated leaves as well (Fig. 1A). This indicated that the transcription of the linearized plasmid clones and the capping of these transcripts occurred normally, and would not account for the lack of productive infection in the soybean and $N$. benthamiana plants (Table 1). The susceptibility of the plants used in the experiments to AIMV infection was tested using wild-type AIMV, which produced clear visible symptoms in inoculated and upper uninoculated leaves of soybean and $N$. benthamiana plants 1 to 4 weeks postinoculation. The status of these AlMV-infected plants was confirmed by western blots at 8 (Fig. 1B) and 15 days postinoculation (data not shown).

We had no difficulties in finding laboratory records of the soybean infection with the wild-type AIMV; however, we could not find any record of soybean inoculation with the transcripts of the recombinant cDNAs of AIMV RNAs 1 and 2 and the recombinant Rg24-containing gene expressed from RNA3 described in the text and in Figure 6 . Therefore, we are unable to critically reevaluate those data.

In light of the above findings, we must advise the readers that the results presented in Figure 6 of literature citation 1 and the corresponding text section of that paper are not reproducible in our laboratory and should in our opinion be disregarded. We urge the readers not to rely on the previously reported data.

\section{LITERATURE CITED}

1. Fleysh, N., Deka, D., Drath, M., Koprowski, H., and Yusibov, V. 2001. Pathogenesis of Alfalfa mosaic virus in soybean (Glycine max) and expression of chimeric rabies peptide in virus-infected soybean plants. Phytopathology 91:941-947. 